\title{
Exploração de fatores de risco para lesões no atletismo de alta performance
}

\author{
Carlos Marcelo Pastre1, Guaracy Carvalho Filho², Henrique Luiz Monteiro³,
} Jayme Netto Júnior ${ }^{4}$, Carlos Roberto Padovani ${ }^{5}$ e Ángel Basas García 6

\section{RESUMO}

Os fatores de risco para instalação de lesões do esporte têm sido pesquisados no sentido de facilitar o entendimento sobre o assunto. Contudo, para altos níveis de performance, nos eventos de pista e campo do atletismo, são escassos os documentos que abordam o tema. Assim, a partir da possibilidade de reunir informações sobre a condição descrita, objetivou-se com o presente estudo a exploração de fatores de risco para lesões desportivas no atletismo, a partir de inquérito aplicado a atletas da elite mundial da modalidade. A população foi composta por 60 homens e 60 mulheres alocados em grupos conforme a especificidade de sua modalidade (velocidade, resistência, arremessos e saltos). Realizou-se entrevista utilizando-se de inquérito de morbidade referida, abordando questões sobre variáveis antropométricas e de treinamento, assim como lesões. Utilizou-se a técnica da análise de variância paramétrica para as variáveis antropométricas (idade, peso, estatura) e da técnica da análise de variância não paramétrica em relação às variáveis de treinamento (anos de treinamento e horas semanais). Para associação entre momento de lesão e especialidades, utilizou-se do teste de Goodman em nível de $5 \%$ de significância. Os resultados mostraram que houve elevada freqüência de lesões na modalidade em ambos os sexos. As taxas de lesão por atleta entrevistado foram de 0,92 (velocidade), 1,08 (resistência), 1,22 (saltos) e 1,20 (arremessos). Não houve diferença estatisticamente significante para as variáveis antropométricas e de treinamento em relação às provas, com exceção dos saltadores, que apresentaram diferenças para estatura e tempo de treinamento; nesse caso, os acometidos são mais altos ou praticam atletismo há menos tempo $(P<0,05)$. Concluiu-se que, para população estudada, o risco de lesão é acentuado, mas sem relação entre variáveis e presença de agravos, salvo para especialistas em provas de saltos, que apresentaram estatura e tempo de treinamento como fatores predisponentes à lesão.

1. Professor Doutor, Departamento de Fisioterapia, Faculdade de Ciências e Tecnologia, UNESP - Presidente Prudente. Fisioterapeuta Consultor Confederação Brasileira de Atletismo.

2. Professor Doutor, Departamento de Ortopedia e Traumatologia, FAMERP, São José do Rio Preto.

3. Professor Doutor, Departamento de Educação Física, Faculdade de Ciências, Unesp - Bauru.

4. Professor Mestre, Departamento de Fisioterapia, Faculdade de Ciências e Tecnologia, Unesp - Presidente Prudente. Técnico da Seleção Brasileira de Atletismo.

5. Professor Titular, Departamento de Bioestatística, Instituto de Biociências, Unesp - Botucatu.

6. Fisioterapeuta da Real Federação Espanhola de Atletismo.

Aceito em 15/7/06.

Endereço para correspondência: Carlos Marcelo Pastre, Rua Fernão Dias, 950, Vila Santa Tereza - 19023-280 - Presidente Prudente, SP, Brasil. Email: pastre@fct.unesp.br \begin{tabular}{ll}
\hline Palavras-chave: & Lesões do esporte. Atletas. Treinamento desportivo. Variáveis an- \\
& tropométricas. \\
Keywords: & Sports injuries. Athletes. Sport training. Anthropometric variables.
\end{tabular}

\section{ABSTRACT \\ Finding of risk factors for injuries in high performance athletics}

The risk factors for installation of sports injuries have been researched to facilitate the understanding about the issue. However, for high performance levels, in the track and field events of athletics, the documents that approach the theme are scarce. Thus, with the possibility to collect information about the described condition, this study aimed the exploration of risk factors for sport injuries in athletics, by inquiring world elite athletes of the modality. The population was composed by 60 men and 60 women allocated in groups according to the specific modality (speed, resistance, throws and jumps). The interviews were made using a morbidity referred inquiry, approaching subjects on anthropometrics and training variables, as well as injuries. The technique of the analysis of parametric variance was used for the anthropometrics variables (age, weight, stature) and of the technique of the no parametric variance analysis in relation to the training variables (years of training and weekly hours). The Goodman's test was used in level of $5 \%$ of significance for the association between injury moment and specialties. The results showed an elevated frequency of injuries in the modality for both genders. The injury taxes for interviewed athlete were 0,92 (speed), 1,08 (resistance), 1,22 (jumps) and 1,20 (throws). There was not significant statistical difference for the anthropometrics variables and of training in relation to the proofs, except to the jumpers, that presented differences for stature and time of training; in that case, injured are the taller or those that practice athletics for less time $(P<$ 0.05). In conclusion, for studied population, the risk of injury is accentuated, but without relationship between variables and presence of injuries, except for specialists in jumps, that presented stature and time of training as risk factors to the injury.

\section{INTRODUÇÃO}

O atletismo merece destaque por sua importância no contexto histórico esportivo e atenção pela diversidade de eventos na própria modalidade, caracterizados por especificidade na execução gestual e dinâmica na forma de treinamento, que se acentuam em altas performances ${ }^{(1-2)}$.

Tal condição determina a necessidade de enfoques particulares relacionados a cada grupo de eventos atléticos que apresentam características técnicas e exigências fisiológicas semelhantes, para almejar tanto desempenho físico, quanto manutenção da saúde do atleta, assim como a associação dessas condições ${ }^{(3)}$.

Contudo, apesar do avanço das ciências e tecnologia, dados epidemiológicos referentes à modalidade revelam elevada freqüência de lesões com perfis multifatoriais, o que causa preocupação 
aos profissionais envolvidos, principalmente devido ao afastamento das atividades, que interrompe o processo de adaptações orgânicas, influenciando o rendimento e que, em alguns casos, leva à incapacidade permanente para o esporte ${ }^{(4-7)}$

Apesar de a situação-problema ser clara, ainda há escassez de informações sobre as lesões dentro da modalidade. Chalmers ${ }^{(8)}$ destaca que são evidentes as dificuldades ao seu acesso, resultando em descontrole a respeito da realidade de instalações de agravos à saúde dos praticantes e, dessa forma, comprometendo os processos de quantificação, identificação de fatores causais e, principalmente, adequação de métodos preventivos.

Dessa forma, investigar a ocorrência de lesões e relacioná-las às características individuais dos atletas, como variáveis antropométricas e sua exposição ao treinamento, pode contribuir no processo de entendimento dos possíveis fatores causais para ocorrência de agravos, sobretudo em altos níveis de performance.

Assim, constituiu-se como objetivo da presente pesquisa o estudo da associação de variáveis como peso, idade, estatura e tempo de treinamento à presença de lesões desportivas, a partir de inquérito aplicado a atletas da elite mundial do atletismo.

\section{MÉTODOS}

\section{População de estudo}

Para realização da pesquisa utilizaram-se como população de estudo 120 atletas de alto rendimento, de vários países, especialistas em diferentes provas, participantes de campeonatos internacionais, promovidos pela Federação Internacional de Atletismo Amador, sendo 60 homens e 60 mulheres, 36 especialistas em provas de velocidade, 36 em provas de resistência, 18 em saltos e 30 em arremessos.

Os mesmos foram selecionados à sorte, mas garantiu-se equilíbrio nas alocações entre os sexos e provas, e representaram, aproximadamente $10 \%$ do universo possível para coleta. A média da idade dos atletas foi de 26,28 \pm 4,36 anos, o peso de 71,85 \pm $19,34 \mathrm{~kg}$ e estatura de 1,76 $\pm 0,11 \mathrm{~m}$. Em relação ao tempo de prática, a média foi de 11,81 $\pm 4,75$ anos de treinamento e de $23,07 \pm 6,71$ horas semanais.

\section{Delineamento observacional}

A coleta de dados foi realizada durante competições promovida pela Federação Internacional de Atletismo, como Meetings Internacionais e Campeonato Mundial, com participação da Confederação Brasileira de Atletismo. Por se tratar de eventos importantes para os participantes, a abordagem para coleta de informações foi feita em momentos distantes do período de preparação para as provas de cada atleta, como durante sua presença nas áreas de lazer, arquibancadas da pista de aquecimento ou competição e refeitório, conforme o sugerido por Pastre et al..(7).

\section{Técnicas e procedimentos de campo}

Os dados utilizados para execução do trabalho foram obtidos por meio de inquérito de morbidade referida, a partir da elaboração de um questionário específico baseado em vivências práticas com a modalidade. $\mathrm{O}$ instrumento de coleta foi previamente testado.

Houve tradução do instrumento para língua inglesa para padronizá-lo aos entrevistadores. Três profissionais da área de saúde, fluentes em inglês, francês espanhol, português e italiano nos termos técnicos do estudo e, com experiência de trabalho em suas respectivas seleções nacionais (Brasil, Espanha e Cuba), após discussão e concordância sobre as questões contidas no instrumento, coletaram e registraram os dados.

Assim, a abordagem para convite à participação e os relatos da pesquisa foram possíveis em cinco idiomas, que compreendiam a maioria dos atletas abordados. Ainda que o atleta não compreendesse os idiomas oferecidos para realização do estudo, sempre havia, em sua equipe, alguém fluente em pelo menos um deles e, assim, auxiliava no processo de tradução. Segundo Almeida Filho e Rouquayrol(9), tal auxílio, na falta de condições ou entendimento, é válido para coleta na forma de inquérito.

\section{Descrição do inquérito de morbidade}

$O$ instrumento continha questões sobre dados pessoais relativos aos atletas, como: sexo, idade, peso, estatura e tempo de treinamento em anos e em horas por semana, além da prova em que é especialista. Para obtenção das informações referentes à lesão desportiva, foram inseridas apenas duas questões que definiam presença ou ausência, além do momento em que ocorreu, ou seja, treinamento ou competição.

Destaca-se que o instrumento utilizado para coletar os dados do estudo foi baseado no inquérito validado e descrito por Pastre et al. ${ }^{(7)}$ como fidedigno para registrar informações sobre lesões desportivas nessa modalidade, sobretudo para o desenho metodológico proposto para a presente pesquisa, que define a busca de lesões dentro de uma temporada completa de treinamentos e competições.

Para efeito de estudo, considerou-se lesão desportiva (LD) qualquer dor ou afecção músculo-esquelética resultante de treinamentos e competições desportivas e que foi suficiente para causar alterações no treinamento normal, seja na forma, duração, intensidade ou freqüência, conforme já utilizado em outras pesquisas ${ }^{(10)}$.

\section{Organização e descrição das categorias das variáveis}

No sentido de facilitar a análise e apresentação dos resultados, as categorias ou subdivisões das variáveis, já descritas, foram agrupadas em blocos mais expressivos de resultados, sem, no entanto, modificar a essência de sua origem ou as conclusões do estudo.

Inicialmente, foi destacada a variável prova, para esclarecer as características próprias de cada participante. Entende-se como "velocidade" o conjunto de provas que compreendem 100, 200 e 400 metros rasos, 100, 110 e 400 metros com barreiras; seus praticantes são denominados velocistas. Para as provas de "resistência", foram considerados especialistas os que disputavam $800,1.500,5.000$ e 10.000 metros rasos, também chamados de meio-fundistas ou fundistas. Nas provas de "saltos", estavam contidos participantes de salto em distância, triplo e em altura. E, por fim, as provas de "arremessos" continham participantes de arremesso de peso, lançamento de disco e dardo. Tal classificação é adotada pela Federação Internacional de Atletismo, sendo modificada e utilizada por Laurino et al.(11) em sua pesquisa a partir de praticantes da modalidade.

O momento da lesão foi caracterizado apenas por duas categorias, o treinamento e a competição. A primeira compreendia as situações de prática de atividades durante períodos de preparação, geral, específico e pré-competitivo. A segunda apenas destacava o momento de competição em eventos de âmbito regional, nacional ou internacional.

As variáveis antropométricas e de treinamento foram eleitas como possíveis fatores de risco para instalação de lesões. Optouse por levantar questões sobre idade, peso, estatura, além de anos de treinamento e horas semanais, traçando perfil dos sujeitos que sofreram ou não lesões, para identificar algumas das possíveis causas intrínsecas ou extrínsecas descritas na literatura.

\section{Aspectos legais da pesquisa}

O tema da pesquisa foi encaminhado ao Comitê de Ética em Pesquisa da Faculdade de Medicina de São José do Rio Preto e o início das coletas começou após sua análise e aprovação. Todos os participantes consentiram em integrar a população de estudo após leitura e compreensão de termo de consentimento livre e esclarecido, autorizando por escrito a utilização das informações cedidas. 


\section{Procedimentos estatísticos}

A abordagem descritiva dos achados foi realizada por meio da distribuição de freqüências absolutas e relativas de ocorrência de relatos, assim como pelo cálculo de taxas relativas à razão entre eventos e participantes, no sentido de acrescentar elementos de quantificação à literatura sobre o tema.

Para o estudo analítico comparativo entre atletas acometidos e não acometidos por lesões, optou-se pela utilização da técnica da análise de variância paramétrica para as variáveis antropométricas (idade, peso, estatura) e da técnica da análise de variância não paramétrica em relação às variáveis de treinamento (anos de treinamento e horas semanais).

Em relação à associação entre momento de lesão e especialidades, utilizou-se do teste de Goodman para análise dentro e entre populações multinomiais. Nos casos de análise ou associação, todas as discussões dos resultados foram realizadas para nível de $5 \%$ de significância.

\section{RESULTADOS}

A tabela 1 mostra as freqüências de distribuição dos participantes e de relatos e taxas de lesões segundo o sexo. Foi notado que os valores anotados são bastante próximos quando comparados os gêneros. Além disso, observou-se elevado número de ocorrências e taxas de lesões para o universo pesquisado.

\section{TABELA 1}

Distribuição dos atletas quanto à presença ou ausência de lesões, freqüência de lesões relatadas e taxas de lesões, segundo o sexo

\begin{tabular}{llcc}
\hline \multirow{2}{*}{ Variáveis } & Categoria das variáveis & \multicolumn{2}{c}{ Sexo } \\
\cline { 3 - 4 } & & Masculino & Feminino \\
Atletas participantes & Lesionados & $40(48,2 \%)$ & $43(51,8 \%)$ \\
& Não lesionados & $20(54,1 \%)$ & $17(45,9 \%)$ \\
& Total & $60(50,0 \%)$ & $60(50,0 \%)$ \\
\hline Lesões relatadas & Total & $65(50,0 \%)$ & $65(50,0 \%)$ \\
\hline Taxas de lesão & Participante & 1,08 & 1,08 \\
& Atleta lesionado & 1,62 & 1,51 \\
\hline
\end{tabular}

Nota: A taxa de lesão por praticante é obtida pela divisão do número total de lesões pelo número total de atletas entrevistados e a taxa de lesão por atleta lesionado é igual ao número total de lesões dividido pelo total de atletas lesionados.

São apresentadas na tabela 2 a distribuição dos atletas na presença ou ausência de agravos e as taxas de lesões, ambas segundo a prova. Observa-se que para todas as provas houve maior número de atletas acometidos, destacando-se as de saltos em valores relativos. Quando a investigação refere-se apenas aos acometidos, os destaques são as provas de resistência e arremessos. No total, a maioria dos entrevistados referiu ter sofrido lesões durante a temporada.

\begin{tabular}{|c|c|c|c|c|}
\hline \multicolumn{5}{|c|}{$\begin{array}{c}\text { TABELA } 2 \\
\text { Distribuição dos atletas quanto à presença ou } \\
\text { ausência de agravos e taxas de lesões, segundo a prova }\end{array}$} \\
\hline \multirow[t]{2}{*}{ Prova } & \multicolumn{2}{|c|}{ Atleta } & \multicolumn{2}{|c|}{ Taxas de lesão } \\
\hline & Lesionado & Não lesionado & Participante & Atleta lesionado \\
\hline Velocidade & $24(66,67 \%)$ & $12(33,33 \%)$ & 0,92 & 1,37 \\
\hline Resistência & $22(61,11 \%)$ & $14(38,89 \%)$ & 1,08 & 1,77 \\
\hline Saltos & $15(83,33 \%)$ & $3(16,67 \%)$ & 1,22 & 1,46 \\
\hline Arremessos & $22(73,33 \%)$ & $8(26,67 \%)$ & 1,20 & 1,63 \\
\hline Total & $83(69,17 \%)$ & $37(30,83 \%)$ & 1,08 & 1,56 \\
\hline
\end{tabular}

Nota: A taxa de lesão por praticante é obtida a partir do número total de lesões relatadas dividido pelo total de atletas em cada prova e a taxa de lesão por atleta lesionado é obtida a partir da divisão entre o total de lesões relatadas pelo total de atletas lesionados em cada prova.
A tabela 3 mostra a comparação do perfil antropométrico dos atletas acometidos ou não por lesões segundo cada prova. Nas variáveis idade e peso, não houve diferença estatisticamente significante a partir das análises dentro das provas entre acometidos ou não. Todavia, para a variável estatura, notou-se que, apenas dentro do grupo de saltadores, sujeitos com maior estatura são mais predispostos a apresentar lesões $(P<0,05)$.

TABELA 3

Distribuição dos valores de média e desvio-padrão das variáveis antropométricas dos participantes, segundo presença ou ausência de lesão e tipo de prova

\begin{tabular}{llcccc}
\hline \multirow{2}{*}{ Variável } & \multirow{2}{*}{ Lesão } & \multicolumn{4}{c}{ Prova } \\
\cline { 3 - 6 } & & Velocidade & Resistência & Saltos & Arremessos \\
\hline \multirow{2}{*}{ Idade } & Ausente & $25,6 \pm 4,5$ & $27,8 \pm 5,2$ & $25,7 \pm 2,5$ & $25,0 \pm 3,2$ \\
& Presente & $25,8 \pm 3,2$ & $28,7 \pm 5,2$ & $24,1 \pm 4,1$ & $26,0 \pm 3,9$ \\
\hline Peso & Ausente & $68,8 \pm 9,0$ & $58,1 \pm 9,3$ & $66,7 \pm 10,0$ & $101,7 \pm 10,3$ \\
& Presente & $65,5 \pm 11,8$ & $57,1 \pm 7,6$ & $73,5 \pm 13,9$ & $92,4 \pm 20,2$ \\
\hline Estatura* & Ausente & $1,76 \pm 0,08$ & $1,71 \pm 0,11$ & $1,71 \pm 0,10 a$ & $1,81 \pm 0,06$ \\
& Presente & $1,72 \pm 0,11$ & $1,71 \pm 0,08$ & $1,83 \pm 0,11 \mathrm{~b}$ & $1,81 \pm 0,10$ \\
\hline
\end{tabular}

* variável com diferença estatística observada para o grupo de saltos $(P<0,05)$, sendo $a<b$.

Em relação às variáveis de treinamento (anos de treino e horas semanais) e sua associação com instalação de lesões, observa-se na tabela 4, a partir dos achados, que dentro das provas de saltos, sujeitos que não são acometidos por lesões treinam há mais tempo em relação aos que referiram lesões $(P<0,05)$. Para as outras especialidades e variável horas semanais, as distribuições foram casuais, ou seja, sem diferença significante.

TABELA 4

Distribuição dos valores da mediana das variáveis de treinamento dos participantes, segundo presença ou ausência de lesão e tipo de prova

\begin{tabular}{|c|c|c|c|c|c|}
\hline \multirow[t]{2}{*}{ Variável } & \multirow[t]{2}{*}{ Lesão } & \multicolumn{4}{|c|}{ Prova } \\
\hline & & Velocidade & Resistência & Saltos & Arremessos \\
\hline \multirow{2}{*}{$\begin{array}{l}\text { Anos de* } \\
\text { treinamento }\end{array}$} & Ausente & 8 & 12 & $19 b$ & 9 \\
\hline & Presente & 10 & 14 & $11 a$ & 12 \\
\hline \multirow[t]{2}{*}{ Horas semanais } & Ausente & 16 & 26 & 20 & 21 \\
\hline & Presente & 20 & 24 & 24 & 28 \\
\hline
\end{tabular}

* variável com diferença estatística observada para o grupo de saltos $(P<0,05)$, sendo $a<b$.

Na tabela 5 é apresentada a distribuição de freqüências do momento de lesão segundo a prova. Nota-se que não há diferença entre as provas em relação a cada momento. Contudo, para todas as especialidades é destacada a ocorrência da maioria das lesões nas sessões de treinamento quando comparadas com as competições.

TABELA 5

Distribuição percentual dos relatos do momento da lesão segundo a prova

\begin{tabular}{lrrr}
\hline \multirow{2}{*}{ Prova } & \multicolumn{3}{c}{ Momento } \\
\cline { 2 - 4 } & Treinamento & Competição & Total \\
Velocidade & $81,81 \mathrm{aB}$ & $18,19 \mathrm{aA}$ & 33 \\
Resistência & $94,87 \mathrm{aB}$ & $5,13 \mathrm{aA}$ & 39 \\
Saltos & $77,27 \mathrm{aB}$ & $22,73 \mathrm{aA}$ & 22 \\
Arremessos ou lançamentos & $86,11 \mathrm{aB}$ & $13,09 \mathrm{aA}$ & 36 \\
\hline
\end{tabular}

Nota: Teste de Goodman para contrastes entre e dentro de populações multinomiais. Diferença estatisticamente significante dentro de cada prova $(P<0,05)$ entre os momentos, sendo $A<B$. Entre as provas para cada momento, não houve diferença significante, sendo $\mathrm{a}=\mathrm{a}$. 


\section{DISCUSSÃO}

A elevada freqüência de distribuição de lesões observada na presente pesquisa, acometendo 69,17\% dos atletas entrevistados, e as altas taxas de lesões por atleta refletem o importante risco de instalação de agravos originados pela prática do atletismo, especificamente em alta performance. Esses resultados estão próximos dos apresentados por D'Souza ${ }^{(2)}$ (61,2\%), Bennell e Crossley(10) $(76 \%)$ e Laurino et al.(11) $(76,7 \%)$ em estudos epidemiológicos.

Watson e DiMartino(12) notaram menor número de freqüência de agravos em relação ao que descreve a maioria dos trabalhos sobre lesões nos esportes, porém, o período de observação da população investigada foi consideravelmente menor (77 dias) que o utilizado nas demais pesquisas citadas, que variavam entre oito e 12 meses.

Sobre a distribuição de agravos segundo o sexo, os achados desta pesquisa são semelhante aos observados por McKay et al.(13), Baumhauer et al.(14), Beachy et al.(15) e Dane et al.(16), que não observaram diferenças na distribuição de lesões entre gêneros. Por outro lado, pesquisas mostraram maior tendência para instalação de LD em homens, como no caso de Messina et al. ${ }^{(17)}$ e Stevenson et al. ${ }^{(18)}$, e houve outras que concluíram que mulheres apresentavam maior predisposição aos agravos, conforme observado por Gwinn et al.(19) e Bell et al.(20). Em síntese, as hipóteses levantadas pelos autores para explicar as diferenças referiam-se às variações anatômicas e hormonais entre sexos e não às causas químicas ou biomecânicas. Pela escassez de estudos com análises detalhadas sobre tais aspectos, também sugeriram investigações aprofundadas sobre o tema.

Em relação à idade e ocorrências de LD, Soderman et al.(21) e Wiesler et al.(22) encontraram resultados condizentes com os verificados nesta pesquisa, ou seja, em seus estudos não notaram associação entre tal fator e a presença de agravos. No entanto, para Murphy et al.(23), em artigo de revisão sobre fatores de risco para extremidades inferiores, atletas mais velhos seriam mais predispostos, por ser submetidos ao estresse, volume ou intensidade de treinamento por período de tempo maior em relação aos mais novos.

Para as variáveis antropométricas, na maioria das observações entre as modalidades e dentro delas, não houve associação entre presença ou ausência de lesões e perfil populacional, conforme também descreveram Wiesler et al.(22), Baumhauer et al..(14) e Bennell et al. ${ }^{(24)}$ em investigações envolvendo lesões e atletas.

Todavia, para os saltadores, os grupos de atletas lesionados apresentaram perfil de altura, estipulado pela média, significantemente maior quando comparados com os não lesionados, fato que Netto Jr. ${ }^{(25)}$ também constatou em seu estudo, que explorou lesões musculares em atletas olímpicos praticantes de atletismo. Para modalidade estudada, não foram encontrados registros que indicassem o contrário do que foi verificado. Contudo, Orchard e Seward(26), analisando lesões em jogadores de futebol americano, notaram o inverso, ou seja, maior predisposição de lesões em indivíduos mais baixos.

As razões exatas para ocorrência desses fatos não são definidas na literatura revisada, mas uma hipótese levantada para explicá-las poderia estar relacionada a aspectos biomecânicos. Nesse sentido, Zatsiorsky ${ }^{(27)}$ e Whiting e Zernicke ${ }^{(28)}$ destacam que, nos saltos, a magnitude de força aplicada aos tecidos do corpo pode ser considerável, principalmente quando movimentos são executados excessivamente ou com deficiência coordenativa e, dessa forma, apresentando vetor resultante direcionado às inserções musculares ou articulações, favorecendo a instalação de lesões, e que diferentes braços de alavanca, determinados por biótipos, podem representar diferentes possibilidades de distribuição de cargas.

Outro aspecto considerado como fator causal de lesão, mas no âmbito extrínseco, diz respeito à exposição do atleta à prática es- portiva ou quantidade de trabalho realizado. Para este estudo, não se observou relação significante entre ocorrência de lesão e perfil de exposição ao treinamento, com exceção para os saltadores. Esses resultados concordam com os achados de D'Souza(2), que não percebeu diferença entre grupos de lesionados ou não. Contudo, Marti et al.(29) e Lysholm e Wiklander ${ }^{(30)}$ observaram relação direta entre exposição ao treinamento e instalação de lesões em suas pesquisas, ou seja, quanto maior o tempo de prática esportiva, ou quantidade de trabalho realizado, mais elevado o risco de instalação de LD.

Discutindo apenas a exceção, especialistas em provas de saltos mostraram tendência maior à lesão nos que praticam atletismo há menos tempo. Como hipótese para explicar tal fato, podese sugerir o fator experiência, sobretudo pela implicação técnica inerente à modalidade, conforme descrevem Whiting e Zernicke ${ }^{(28)}$. Porém, apesar das possíveis comparações e hipóteses, deve-se considerar como limitação do estudo o reduzido número de sujeitos não lesionados, para extrapolação dos achados, principalmente na apresentação das medidas descritivas dessa variável, para sugeri-las como fator predisponente às lesões.

Além do descrito, foram explorados dados sobre o momento de ocorrência de lesão, destacando o treinamento como sendo o período mais propício à instalação de agravos. Condição semeIhante foi observada por D'Souza(2) e Pastre et al. ${ }^{(7)}$ em suas investigações. Uma explicação simplista para o fato poderia estar relacionada ao tempo de exposição às atividades, em que o treinamento se apresenta de forma mais evidente em relação ao volume. Durante o treinamento, o principal objetivo é aumentar os níveis de possibilidades funcionais do organismo e das qualidades físicas necessárias para o desporto, assim como desenvolver os atos motores específicos, segundo Barbanti et al.(31), Rhea et al.(32), nesse sentido, possibilita maior exposição do atleta às condições causadoras de lesão.

Apesar dos achados, por não ser possível um fator de correção sobre o tempo de exposição às práticas esportivas (treino ou competição) pelo teor das informações registradas, afirmar que o treinamento é o principal momento para instalação pode ser precipitado, representando outra limitação do estudo, principalmente porque os níveis de estresse físico e mental, além da necessidade óbvia de performance, são mais evidentes durante as sessões de competições.

Como principais limitações do estudo, além do já discutido referente às variáveis, estatura e tempo de treinamento para saltadores e momento da lesão para todos os grupos de provas, destacam-se as reduzidas possibilidades de comparação dos resultados com outros disponíveis na literatura, não só pelo pequeno número de pesquisas encontradas relacionadas à população e ao tema adotados neste trabalho, mas também pela falta de padronização nos registros de informações referentes às lesões. Tais condições impossibilitam uma discussão mais aprofundada sobre o assunto e sugerem adoção de metodologias semelhantes para coleta de dados no sentido de facilitar futuras comparações.

Por fim, destaca-se que os achados do presente estudo somamse à escassa literatura referente às lesões no atletismo de alta performance, em particular ao contexto mundial que foi abordado. Além disso, pela experiência, a pesquisa aponta o instrumento e os métodos de coleta como facilitadores de processos de quantificação de lesões e exploração de fatores de risco para sua instalação em populações semelhantes, favorecendo acompanhamentos longitudinais e até testes de eficiência para programas preventivos.

\section{CONCLUSÕES}

A partir dos achados, pode-se concluir que é elevado o risco para instalação de lesões em praticantes de atletismo de alta performance, sem associação entre variáveis antropométricas ou tem- 
po de treinamento e ocorrência de agravos, com exceção para estatura e tempo de prática esportiva para os especialistas em provas de saltos.

Todos os autores declararam não haver qualquer potencial conflito de interesses referente a este artigo.

\section{REFERÊNCIAS}

1. Nattiv A. Stress fractures and bone health in track and field athletes. J Sci Med Sport. 2000;3:268-79.

2. D'Souza D. Track and field athletics injuries - A one year survey. Br J Sports Med. 1994;28:197-202.

3. Shiffer J. Overuse injuries of the Achilles tendon. New studies in athletics. 2003; 18:65-106.

4. Kettunen JA, Kujala UM, Kaprio J, Koskenvuo M, Sarna S. Lower-limb function among former elite male athletes. Am J Sports Med. 2001;29:2-8.

5. Bahr R. Recent advances: sports medicine. BMJ. 2001;223:328-31.

6. Bahr R, Holme I. Risk factors for sports injuries - A methodological approach. $\mathrm{Br}$ J Sports Med. 2003;37:384-92.

7. Pastre CM, Carvalho Filho G, Monteiro HL, Netto Jr J, Padovani CR. Lesões desportivas no atletismo: comparação entre informações obtidas em prontuários e inquéritos de morbidade referida. Rev Bras Med Esporte. 2004;10:1-8.

8. Chalmers DJ. Injury prevention in sport: not yet part of the game? Inj Prev. 2002 8:22-5.

9. Almeida Filho N, Rouquayrol ZM. Introdução à epidemiologia. 3a ed. Rio de Janeiro: Medsi, 2002

10. Bennell KL, Crossley K. Musculoskeletal injuries in track and field: incidence, distribuition and risck factors. Australian Journal of Science and Medicine in Sport. 1996;28:69-75.

11. Laurino CFS, Lopes AD, Mano KS, Cohen M, Abdalla RJ. Lesões músculo-esqueléticas no atletismo. Rev Bras Ortop. 2000;35:364-8.

12. Watson MD, Di Martino PP. Incidence of injuries in high school track and field athletes and its relation to performance ability. Am J Sports Med. 1987;15:2514.

13. McKay GD, Goldie PA, Payne WR. Ankle injuries in basketball: injury rate and risk factors. Br J Sports Med. 2001;35:103-8.

14. Baumhauer JF, Alosa DM, Renstrom AF. A prospective study of ankle injury risk factors. Am J Sports Med. 1995;23:564-70.
15. Beachy G, Akau CK, Martinson M. High school sports injuries. A longitudinal study at Punahou School: 1988 to 1996. Am J Sports Med. 1997;25:675-81.

16. Dane S, Süleyman C, Gürsoy R, Ezirmik N. Sport injuries: relations to sex, sport, injured body region. Percept Mot Skills. 2004;98:519-24.

17. Messina DF, Farney WC, DeLee JC. The incidence of injury in Texas high school basketball. A prospective study among male and female athletes. Am J Sports Med. 1999;27:294-9.

18. Stevenson MR, Hamer P, Finch CF. Sport, age, and sex specific incidence of sports injuries in Western Australia. Br J Sports Med. 2000;34:188-94.

19. Gwinn DE, Wilckens JH, McDevitt ER. The relative incidence of anterior cruciate ligament injury in men and women at the United States Naval Academy. Am J Sports Med. 2000;28:98-102.

20. Bell NS, Mangione TW, Hemenway D. High injury rates among female Army trainees: a function of gender? Am J Prev Med. 2000;18:141-6.

21. Soderman K, Alfredson H, Pietila T. Risk factors for leg injuries in female soccer players: a prospective investigation during outdoor season. Knee Surg Sports Traumatol Arthrosc. 2001;9:313-21

22. Wiesler ER, Hunter DM, Martin DF. Ankle flexibility and injury patterns in dancers. Am J Sports Med. 1996;24:754-7.

23. Murphy DF, Connolly DAJ, Beynnon. Risk factors for lower extremity injury: a review of the literature. Br J Sports Med. 2003;37:13-29.

24. Bennell KL, Malcolm SA, Thomas SA, Reid SJ, Brukner PD, Ebeling PR, et al. Risk factors for stress fractures in track and field athletes. A twelve month prospective study. Am J Sports Med. 1996;24:810-8.

25. Netto Jr J. Lesão muscular: estudo a partir da equipe brasileira de atletismo que participou dos jogos olímpicos de Atlanta-1996. [Dissertação]. Campinas. Unicamp; Faculdade de Educação Física; 2000.

26. Orchard J, Seward H. Epidemiology of injuries in the Australian football league, seasons 1997-2000. Br J Sports Med. 2002;36:39-45.

27. Zatsiorsky VM. Biomecânica no esporte. Performance do desempenho e prevenção de lesão. Rio de Janeiro: Guanabara Koogan, 2004

28. Whiting WC, Zernicke RF. Biomecânica da lesão músculo-esquelética. Rio de Janeiro: Guanabara Koogan; 2001.

29. Marti B, Vader JP, Minder CE, Abelin T. On the epidemiology of running injuries. The 1984 Bern Grand-Prix study. Am J Sports Med. 1988;16:285-94.

30. Lysholm J, Wiklander J. Injuries in runners. Am J Sports Med. 1987;15:168-71.

31. Barbanti VJ, Tricoli V, Ugrinowitsch C. Relevância do conhecimento científico na prática do treinamento físico. Rev Paul Educ Fis. 2004;18:101-9.

32. Rhea MR, Phillips WT, Burkett LN, Stone WJ, Ball SD, Alvae B, et al. A comparison of linear and daily undulating periodized programs with equated volume and intensity for local muscular endurance. Journal of Strength and Conditioning Research. 2003:17:82-7. 